\title{
Chemical Imaging of Biofilms: The Integration of Synchrotron Imaging, Electron Microscopy and Nuclear Magnetic Resonance (NMR) Technologies
}

\author{
M.J. Marshall ${ }^{1}$, S.M. Belchik ${ }^{1}$, A.E. Tucker ${ }^{1}$ W.B. Chrisler $^{1}$ M. Thomas ${ }^{1}$, R.S. Renslow ${ }^{1}$, A.P. Kuprat ${ }^{1}$, A.C. \\ Dohnalkova $^{1}$ and C.J. Hirschmugl ${ }^{2}$ \\ ${ }^{1}$ Pacific Northwest National Laboratory, Richland, Washington 99354 USA \\ ${ }^{2}$ Synchrotron Radiation Center, Stoughton, WI 53589 USA
}

Direct examination of natural and engineered environments has revealed that the majority of microorganisms in these systems live in structured communities termed biofilms. In addition to microbial cells, biofilms are comprised of a poorly characterized organic matrix commonly referred to as extracellular polymeric substance (EPS) that may play roles in facilitating microbial interactions and biogeochemical reactions including extracellular electron transfer. Using highresolution electron microscopy imaging, we have shown copious amounts of highly hydrated bacterial EPS to be produced during microbial metal reduction [1,2]. The juxtaposition of extracellular electron transfer proteins and nanoparticulate reduced metal suggested that EPS played a key role in metal capture and precipitation. Determining the chemical composition of biofilm-associated EPS and understanding how it functions and interacts with inorganic substrates including metal ions and mineral surfaces is needed to connect the molecular-scale biogeochemical processes to those at the microorganism-level, and provide insight to how microbes influence larger, pore-scale biogeochemical reactions.

A multi-faceted, multi-scale approach using a combination of synchrotron-based x-ray and infrared imaging, electron microscopy (EM), non-invasive nuclear magnetic resonance (NMR) imaging will yield a high spatial resolution, chemical image of a biofilm community in its nearest-to-native state as it influences biogeochemical reactions such as the redox-transformation of multivalent metals. For pore- and/or community-scale biofilm investigations in complex environments, x-ray microtomography produced three-dimensional renderings of hydrated Shewanella biofilms and visualized a delicate internal structure and network of pores within biofilms. These pores (e.g., fluid perfusion channels) indicate regions where water, nutrients, or electrolytes can flow through biofilm biomass and may influence pore-scale biogeochemical reactions. We are now computing the distribution of biomass to demonstrate the relative importance of nutrient advection and diffusion for sustenance across the biomass. Non-invasive nuclear magnetic resonance (NMR) imaging will test our predictions of nutrient flow and diffusive properties. We also correlated EM capabilities with scanning transmission X-ray microscopy (STXM) and synchrotron-based Fourier transform-infrared (FTIR) microimaging to produce high-sensitivity, chemical images that correspond to EM images of the biofilm at the nanometer scale. We have developed techniques that facilitate correlative imaging and spectroscopy studies in the absence of chemicals that interfere with native biofilm chemistry. These techniques have been used to collect STXM and FTIR chemical images of biofilms to show spatially resolved chemical gradients within biofilms.

The integration of these techniques will provide detailed, high-resolution visualizations of chemical/elemental information that will strengthen our understanding of how biofilms interact with redox transformable metals to influence local biogeochemical reactions at many scales.

\section{References}

1. Marshall MJ, et al. (2006) PLoS Biol 4: e268.

2. Dohnalkova AC, et al. (2011) Appl Environ Microbiol 77: 1254-1262.

3. Acknowledgment: A portion of this research was performed using the Environmental Molecular Sciences Laboratory (EMSL), a national scientific user facility sponsored by the DOE's Office of Biological and Environmental Research located at PNNL. Battelle Memorial Institute operates PNNL for the DOE under contract DE-AC05-76RL01830. 

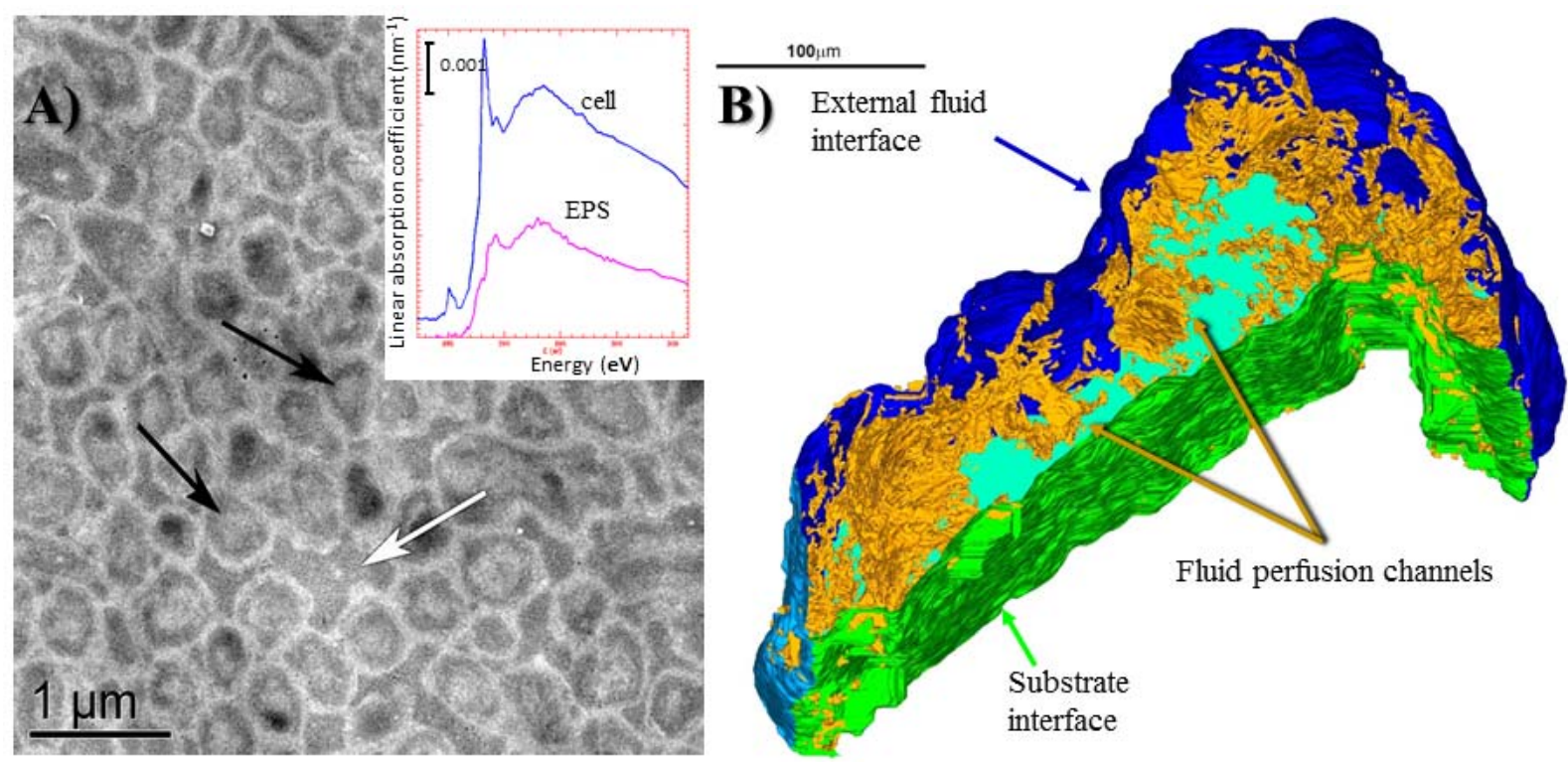

Fig 1. (A) Transmission EM Shewanella biofilm showing dense packaging of cells (black arrows) in extracellular polymeric substance (EPS) matrix (white arrow). Cells were prepared by cryo-sectioning and imaged at room temperature followed by STXM chemical imaging of carbon at the Advanced Light Source (ALS), sector 5.3.2.1 (inset). (B) Segmentation $(\sim 250 \mu \mathrm{m}$ thick $[\mathrm{z}$ direction]) of $\mathrm{x}$-ray microtomography dataset provided a detailed visualization of water channel interconnectivity within hydrated Shewanella biofilm. Using segmented fluid perfusion channels and the biofilm external surfaces; we are computing the distribution of biomass as a function of distance from the nearest point of nutrient advection. Data collected at the Advanced Photon Source (APS), sector 2BM. 